\title{
Adaptive Image Steganalysis: Adaptive Image Segmentation using Enhanced Canny Edge Detection Algorithm
}

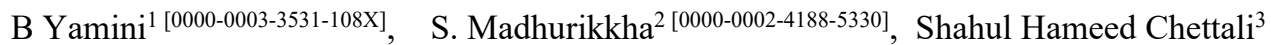 \\ [0000-0002-9761-0432], Mercy Theresa $\mathrm{M}^{4}$ [0000-0002-3245-1215], A. Jesudoss 5 [0000-0001-8515-7530] \\ \{yamini.subagani@gmail.com ${ }^{1}$,madhurikkha@jeppiaarcollege.org ${ }^{2}$, in.hameed@gmail.com ${ }^{3}$, \\ mercyjesudossa@gmail.com ${ }^{4}$, jesudossas@gmail.com ${ }^{5}$ \}
}

Assistant Professor, Department of CSE, College of Engineering and Technology, SRM Institute of Science \& Technology (SRMIST), Kattankulathur, Tamil Nadu, India ${ }^{1}$., Assistant Professor, Department of CSE, Jeppiaar Engineering College, Jeppiaar Nagar, Rajiv Gandhi Salai, Chennai, Tamil Nadu, India $^{2}$., Assistant Professor, Institute of CSE, Saveetha School of Engineering, Saveetha Institute of Medical and Technical Sciences, Tamil Nadu, India ${ }^{3}$., Associate Professor, Department of ECE, Prince Shri Venkateswara Padmavathy Engineering College, Chennai, Tamil Nadu, India ${ }^{4}$, Associate Professor, Department of CSE, Sathyabama Institute of Science and Technology, Chennai, Tamil Nadu, India ${ }^{5}$

\begin{abstract}
An expertise method to conceal secret messages in blameless mediums such as videos, audios, texts and digital images by cheating the Human Visual System (HVS) is termed as steganography. Blind Steganalysis is the striking technique to identify, excerpt hidden information from multimedia file with concealed secret information called stego media. To conceal the hidden message, adaptive image steganography determines the exact color adaptive regions or payload locations of the image. Adaptive image steganography's reverse in return is adaptive image steganalysis, in which the secret messages are dig out from the image's color adaptive regions for a familiar or unfamiliar steganographic algorithm that was used for steganography. In the first phase of the adaptive image steganalysis, the adaptive regions of the image are segmented in such a way that the color intensity values of the pixels are compared and grouped to form a region. Enhanced canny edge detection operator outperforms other segmentation algorithms. In the second phase, the statistical features of the pixels are extracted from the spatial domain region using Histogram of Oriented Gradient (HOG) and MatriX Quadtree (MX Quadtree) method. Feature selection from the extracted feature vector subset is done based on distance correlation coefficient method and Markov Random Field (MRF) Cliques method along with a ranking based wrapper approach. In the third phase, classification of images as stego or cover image is done by a binary classifier, Support Vector Machine (SVM). Classification of stego images in the proposed system is done first and then the content extraction of the concealed information from payload locations of the stego images are obtained. This can be achieved by using effective adaptive image segmentation called Enhanced Canny Edge Detection algorithm and feature extraction method. Then, the images are classified by better performing classifier Support Vector Machine as cover or stego images which results in $96.72 \%$ of classification accuracy.
\end{abstract}

Keywords: Adaptive Image Steganalysis, Enhanced Canny Edge Detection, Histogram of Oriented Gradient, MatriX Quad tree, Markov Random Field, Support Vector Machine. 


\section{Introduction}

Statistical pattern recognition approach includes Face recognition, Speech recognition, automatic target recognition and Image classification. Statistical pattern recognition approach in color image steganalysis system has three parts: Image Segmentation, Feature Extraction, Feature Selection and Classifier Selection.

\subsection{Image Segmentation}

Image Segmentation in adaptive image steganalysis renders a most important part in identifying adaptable regions in images by considering the intensity values of the pixel and the pixel value differences in order to group them into a single region from a seed point. Once these adaptive regions are identified clearly in the input image, then it makes the rest of the system works effectively to classify the image as color image or stego image by extracting the hidden messages that are embedded during steganographic process.

\subsection{Feature Extraction}

Feature extraction in image steganalysis task is the key step to analyse the characteristics of the image to identify whether the input image is a cover image or stego image. Principles of feature extraction steps are as follow:

- Discrimination: Features of set of selected adaptive regions of the same image should have knowingly different values.

- Reliability: Features should have similar values which were grouped under a same single region as adaptable region.

- Independence: Features from the adaptive regions should not be strongly correlated to each other which may lead to confusion.

- Optimality: Feature selection, plays a vital role in identification of the stego images. This feature selection results in information rich content for classification.

\subsection{Significance of Feature Selection methods:}

- Enhances classification accuracy of the classifier by dropping the computational difficulty.

- The features should be selected in such a way that it should not be redundant while combining the feature set.

- Decreases the number of features considered for achieving the task efficiently by speeding up the process.

- "Curse-of-Dimensionality" (CoD) problems are addressed by feature selection methods.

- Interpretations of the features that are selected for processing becomes easier.

\subsection{Image Classification}

Classification of the image as cover image or stego image is performed efficiently by appropriate classifier selection. However, no matter, how worthy the applied feature extraction process is, if a poor classification design is implemented and this collapses the whole system. The differentiation between one classification method and another, resides in their ability to identify the stego images exactly. In the training or learning stage, learning methods are categorized as Supervised and Unsupervised learning. 


\section{The State of Art}

The analysis of several literatures discussed in this segment is focused upon several systems for image segmentation, feature extraction, Classification, payload location identification and hidden message extraction process of steganalysis. Deep research analysis has been conducted in expansion of automated steganalysis using color images to support in the field of social networking sites, forensic and defense. Wide-ranging literature analysis has been completed in associated with this research and significant features of relevant contents are defined here. Different techniques have been used in the field of image steganalysis.

\subsection{Image Segmentation Algorithm}

Region identification and segmentation identifies Region of Interest (ROI) during image steganalysis. The different types of Image segmentation methods [1] are discussed in image steganalysis are reviewed for performance. The image segmentation methods are as follows established on: Threshold, Edge, Region, Cluster, Partial Differential Equation and Artificial Neural Network.

\subsection{Threshold Based Image Segmentation.}

The various threshold methods in image segmentation are discussed [2]. According to the application, image segmentation was done by detecting, recognizing and feature measurement. The image was segmented into meaningful regions. Non contextual method called thresholding was used in the gray-scale image to convert it into a binary image. Histogram Dependent Technique (HDT) that uses the thresholding such as global thresholding, local thresholding and adaptive thresholding was analyzed for the performance.

\subsection{Edge Based Image Segmentation.}

The various Edge detection operators for image segmentation are proposed for identifying edges of the image [3]. The authors discussed about Roberts's edge detection for its simplicity with two dimensional array formations of pixels which results in quick response and simplicity compared to other edge detectors. Since two dimensional pixel values were considered for comparison, clear and deep evaluation of pixel values with neighboring pixels were done.

The region identification method using the color planetary intensity is the better way of fining adaptive color regions [4]. The identified regions were used to embed the message, in relevant to intensity of colored region. Image got splitted into three channels on which the canny edge detection was applied for edge mapping and better performance.

\subsection{Region Based Image Segmentation.}

The image segmentation based image steganalysis and the content features of the JPEG images were used to segment the images [5]. Texture complexity of the images was used to create the number of segmented sub images. A perfect classifier was built to classify the sub images as stego images or cover images based on similar texture complexity. Weighted fusion system estimated the better detection accuracy.

\subsection{Cluster Based Image Segmentation.}

A most powerful method called clustering in image segmentation was discussed [6]. The clusters were formed as disjoint sets of an image. Various clustering techniques and its improved versions as k-means, Enhanced k-means, Improved Fuzzy C-Mean algorithm (IFCM) and Fuzzy C-Means (FCM) were proposed. Performance of the grouping methods were analyzed and evaluated using statistical parameters such as Rand Index (RI), Global Consistency Error (GCE), Boundary Displacement Error (BDE) and Variations of information (VOI). Authors concluded that the performance of Enhanced Fuzzy c-means clustering was 
better while compared with other clustering methodologies in terms of convergence rate, performance and accuracy in cluster formation.

\subsection{Watershed Based Image Segmentation.}

The region based approach by using watershed transformation method was discussed in which the gray-scale images were used to perform the watershed approach by flooding process [7]. Watershed transformation method combines the pre-processing and post-processing approach to identify large number of regions for embedding message. The author explained the image processing applications of watershed algorithm that can be applied to gray-scale images, binary images and Texture based image processing.

\subsection{Partial Differential Equation Based Image Segmentation.}

A new method for denoising using Partial Differential Equation (PDE) by an isotropic diffusion technique and total variation technique features were combined and the weighted combinations were obtained [8]. The weighted combinations were used in finding the deviations of the pixels for segment identification. The performance of method resulted in efficient denoising of image by preserving the texture feature and edge data of an isotropic diffusion and total variation methods.

\subsection{Artificial Neural Network Based Image Segmentation.}

There are various methods of Artificial Neural Network (ANN) to detect the hidden information [9]. The Artificial Neural Network methods such as Back Propagation Algorithm (BPA), Functional Update Back Propagation Algorithm (FUBPA) and Radial Basis Function (RBF) were used for steganalysis. Ensemble of back propagation algorithm (BPA) and radial basic function (RBF) called as back propagation algorithm radial basic function (BPARBF) and similarly functional update back propagation algorithm (FUBPA) with radial basic function (RBF) called as Functional Update Back Propagation Algorithm Radial Basic Function (FUBPARBF). The author proved that the ensemble features of FUBPARBF perform better in identifying the hidden information with less number of iterations.

\section{Proposed Work}

This proposed work on the adaptive image steganalysis has three main parts as shown in Figure1. They are image segmentation, adaptive features extraction and Image classification. Detailed framework of proposed system is given in Figure 2.

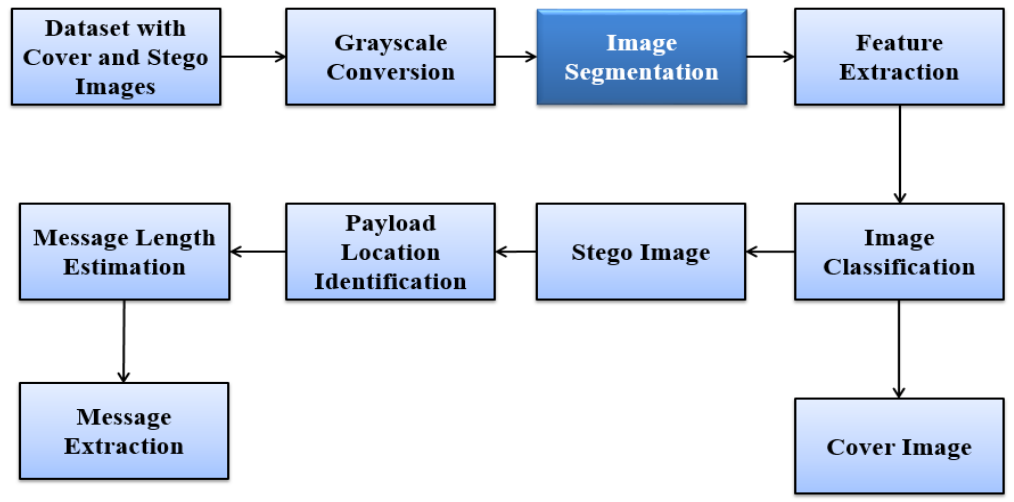

Figure 1: Block Diagram of Proposed System for Adaptive Image Steganalysis 
To generate the dataset of stego images, the standard steganographic algorithms called virtual steganography laboratory (VSL), openstego, outguess and steghide have been applied. The JPEG color images of research interest should be converted to gray scale images. A set of cover and stego images is applied as input to the image segmentation process. Adaptive region of interest is identified using the image segmentation method. The feature vector combinations are extracted from the identified regions using histogram and MX Quadtree approach. Feature selection method called Filter approach and Wrapper approach is used for Feature Subset Generation and Subset Evaluation respectively. This makes the working complexity of the Support Vector Machine Classification very simple and efficient for stego image separation. From the identified stego image, payload location for extracting the hidden message by estimating its length should be identified and measured.

The enhanced wrapper approach works on feature selection from feature combination vector depending on adaptability of features in the region. To improve the performance of the wrapper approach, the classifications based on adaptability of the features are done in the wrapper approach itself. This helps in selecting the appropriate classifier, which is simpler and efficient for the proposed system. From the classified stego images payload locations are identified to extract the hidden messages.

\section{Adaptive Image Segmentation}

In adaptive image steganalysis, there are numerous transforms to be applied on digital images to analyze the images. The first and foremost image processing for adaptive image steganalysis is adaptive region identification by image segmentation. These color images are further used for processing after converting it into its gray scale value. The methods used and analyzed for effective adaptive image segmentation are as follows: 


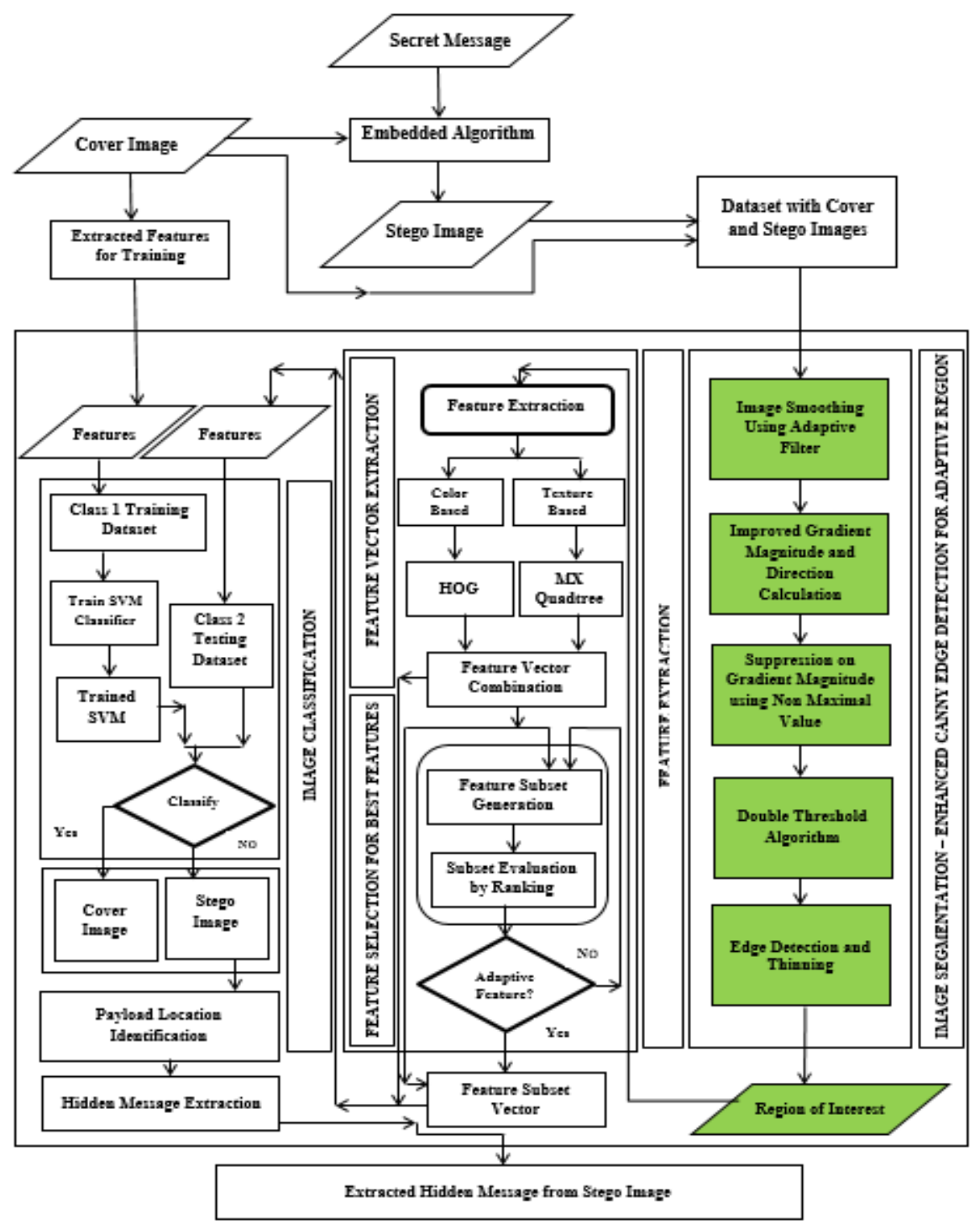

Figure 2: Framework of the Proposed System for Adaptive Image Steganalysis 4.1 Adaptive Region Identification

Homogeneity property of the similar pixels tends these pixels to form a region [10] [11] [12]. The pixels that are grouped under a region imply that they are more similar to the pixels in the same group. The adaptive regions of the image are identified during embedding process to hold maximum capacity of the messages that are to be hidden and the same is more unnoticeable to HVS. The adaptive regions in proposed method are computed by having a seed point probably a pixel and then grouping the pixels that are very similar to the seed pixel. If the neighboring pixel is sufficiently close enough to the seed point, then the pixel belongs to the same region or else to a different region. 
Homogeneity identification and region splitting gets stopped when no more pixels can be added into the group and the adjacent eight regions cannot be merged further. The computational complexity of the proposed adaptive image steganalysis system lies on the initial step called the adaptive region identification by image segmentation. This identification of region helps the feature extraction method to concentrate on a particular region rather than every pixel of the image

\subsection{Edge Detection Algorithm}

The diversity in intenseness of images is marked as edges. Edges are the points that split the images into regions. The regions are separated based on the homogeneity of the pixel's intensity value. This separation of regions helps to define adaptable region of image called interested regions to retrieve the features. In the recommended methodology, adaptable areas are recognized by edges. Geometrical events such as object edges; surface edges and nongeometrical events such as inter reflections; specular shadows are used to identify the edges in an image. Position of the pixel, Intensity level of the pixel, edge directions are described by the edge descriptors. Edges are modeled as edge, ridge, ramp and roof edges by considering its intensity level. Implementation of enhanced canny edge detection algorithm in this work lead to the description of basic methodology named canny edge detection algorithm along with enhanced canny edge detection operator. First, the basic canny edge detection algorithm is explained in detail along with its drawbacks in identification of the edges. Later, implemented concept called enhanced canny edge detection is explained.

\subsection{Canny edge detection algorithm.}

This traditional methodology identifies edges with lower error rate. Over marking of edges are avoided to detect true edge point by avoiding false edge points. Figure 3, defines the step by step process for canny edge detection algorithm

\section{Gaussian Filtering}

Image de-noising is done to categorize the factual edges of the adaptive regions in the image. Gaussian Masking and regular convolution method is used during filtering to smoothen the image. Mask width, assumed for the Gaussian mask determines the reactivity of the noise. Greater the width of the Gaussian mask, lower the noise detection and produces higher error rate. Measured standard deviation $(\sigma)$ value results in sharpness of the intensity transitions. The values of standard deviation $(\sigma)$ are positive real numbers ranges from 0 to 3 .

The Gaussian kernel size of $(2 n+1)^{2}$ is given by the equation (1) in canny edge for the two points $(\mathrm{x}, \mathrm{y})$ and gradient vector is given in equation (2).

$$
G(x, y)=\frac{1}{2 \pi \sigma^{2}} \exp ^{\left[-\frac{\mathrm{x}^{2}+\mathrm{y}^{2}}{2 \sigma^{2}}\right]}
$$

Gradient vector $\nabla G_{v}$ is computed as

$\nabla G_{v}=\left[\begin{array}{l}\partial G_{v} / \partial x \\ \partial G_{v} / \partial y\end{array}\right]$ 


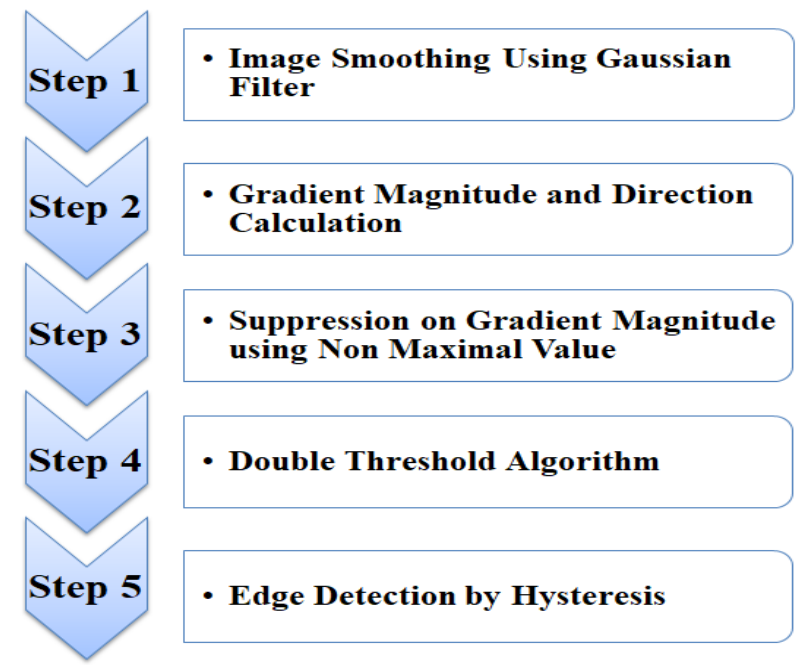

Figure 3: Stepwise Representation of Canny Edge Detection Method

This Gaussian low-pass filter eliminates high incidence components of image and this leads to missing of edges that are weak. Hence, there is a need of an improved filter approach in enhanced canny edge detection algorithm.

Gradient Intensity Comparison degree (Magnitude) and Orientation (Direction) Calculation.

Once the image smoothing is done, the gradient intensity comparison degree and orientation is computed for individual points by first order linear partial differential operator [13]. The partial derivatives of the point $(m, n)$ for any two instances $(i, j)$ are given in equation (3) and (4) as follows,

$$
\begin{gathered}
D_{m}(i, j)=[I(i, j+1)-I(i, j)+I(i+1, j+1)-I(i+1, j)] / 2 \\
D_{n}(i, j)=[I(i, j)-I(i+1, j)+I(i, j+1)-I(i+1, j+1)] / 2
\end{gathered}
$$

The Gradient intensity comparison degree (magnitude) and Gradient orientation (direction) of the point $\mathrm{i}, \mathrm{j}$

$$
\begin{gathered}
M(\mathrm{i}, \mathrm{j})=\sqrt{\left(\left(\mathrm{D}_{\mathrm{m}}{ }^{\wedge} 2(\mathrm{i}, \mathrm{j})+\mathrm{D}_{\mathrm{n}} \wedge 2(\mathrm{j}, \mathrm{j})\right)\right)} \\
\theta(\mathrm{i}, \mathrm{j})=\arctan \left(\mathrm{D}_{\mathrm{m}}(\mathrm{m}, \mathrm{n}) / \mathrm{D}_{\mathrm{n}}(\mathrm{m}, \mathrm{n})\right)
\end{gathered}
$$

$M(\mathrm{i}, \mathrm{j})$ stands for Gradient magnitude in equation (5) and $\theta(\mathrm{i}, \mathrm{j})$ stands for angle direction in equation (6) at $M(\mathrm{i}, \mathrm{j})$. The angle direction represented here is the rounded four angles i.e. Horizontal angle, Vertical angle and two diagonal angles.

The Non Maximal Suppression.

Gradient magnitude and direction have interpolation with respect to canny operator. Canny (edge detection) operators have interruption to gradient intensity comparison (magnitude) and orientation (direction)[13]. The edge point is identified in a manner, when $M$ $(i, j)$ is superior to two subsequent interlude in the orientation of $\theta(i, j)$ at $(i, j)$, if not, then it is treated as non-edge point. Suppression of the gradient magnitude using the non-maximal value is an edge thinning method. This method helps in find the principal edge. Suppression is done on every gradient values except local maximal value. This local maxima value is the accurate change in intensity value. The steps for suppression on gradient magnitude using non-maximal value are given as,

1. Positive gradient and negative gradient directions strength are compared with strength of the current pixel edge. 
2. Subdue value, if strength of current pixel edge is lesser than other considered one else preserves the value.

The directions are given as four angles and they are given by the Table 1,

Table 1 Gradient angle and Direction

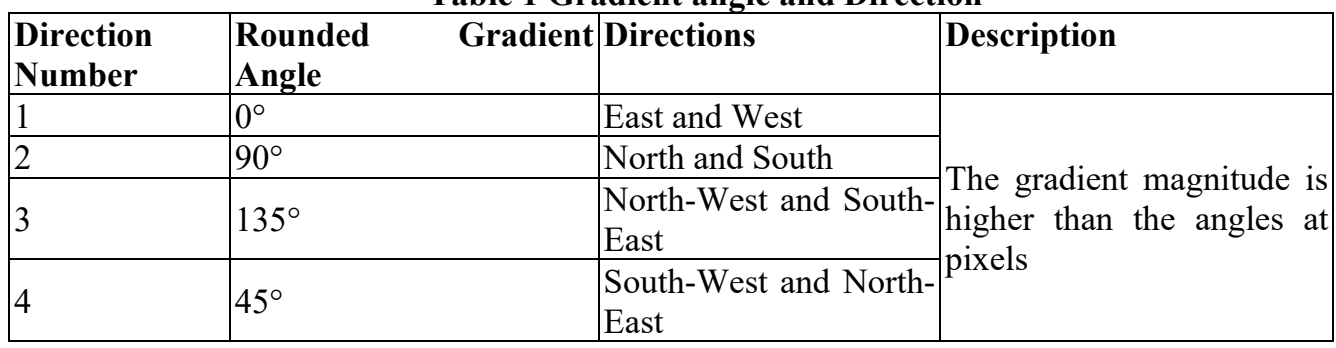

Double Threshold Algorithm.

Two thresholds namely high and low threshold values are selected to filter the fake edge pixels. Strong edge pixels are identified using the gradient pixel's value. When the High threshold is lower than the edge gradient then it is referred as strong edge pixel. Similarly, when the High threshold is higher than the edge gradient and Low threshold is lower than the edge gradient then pixel can be referred as weak edge pixels. Based on the input image, threshold values may vary. Algorithm 1, explains Double threshold algorithm. value.

Input : Suppressed gradient intensity comparison using non-maximal

Method : $\quad$ Selection of two threshold values namely high and low threshold values are used to find the strong or weak edge pixels.

Output : Edge Points

\section{Algorithm 1: Double Threshold Algorithm \\ Step 1: Select the Low Threshold $\left(\mathrm{T}_{1}\right)$ and High Threshold $\left(\mathrm{T}_{\mathrm{h}}\right)$ values. \\ Step 2: Consider a pixel $(\mathrm{i}, \mathrm{j})$ at any point and note the edge and non-edge point. a. if Gradient intensity comparison called magnitude is higher $M(i, j)>T_{h}$, then mark as edge identified point, else if Gradient magnitude $M(i, j)<T_{l}$, then mark as non-edge points \\ b. Pixel positions are marked as suspect point, if and only if when Gradient magnitude $M(i, j)$ value lies in between $T_{h}$ and $T_{1}$.}

Step 3: Mark the Strong edge points.

Edge Detection by Hysteresis.

Strong edge points are the true or final edge points in the image. Usually weak edges are not considered for the edge detection which is eliminated, but in this case analysis is done to evaluate the strength of the weak points that are originally generated by the true edges. The weak edge points and its neighboring 8 pixels are compared to make a decision to preserve this weak point or not. This analysis produces accurate true edge points to be considered.

\subsection{Proposed Enhanced canny edge detection algorithm}

Though detection of edges by canny operator performs better, Major reasons for choosing the enhanced canny operator for edge recognition for recommended system are as follows, 
- Traditional recognition of edge by canny algorithm intended for smoothing noise in the image but it also smooth out the high frequency featured edges. When the edges get smoothen, there raise a problem of breaks in the edge occurrences by leaving the weak point edges.

- Gradient amplitude calculation uses $2 \times 2$ matrixes of pixels as window size to compute finite variance mean value. This computation failed to identify the real edges rather than false edges.

- To find the real edges of different images, different threshold values are needed instead of high threshold value and low threshold value. Thus different threshold values increase the computational complexity of the system.

- Multi-point responses disturb the accuracy of selecting the edge point.

In enhanced canny edge detection weak point edges with high frequency feature are considered to avoid the break in real edges. It uses the adaptive filters for image smoothing. Improved gradient magnitude and direction calculation is done using curvature concept.

Gradient magnitude suppression, Double threshold and edge detection are similar to canny edge detection. Edge thinning is done at last to find the perfect real edge points in the image. Figure 4 and Algorithm 2 shows the step by step process for enhanced canny edge detection Method.

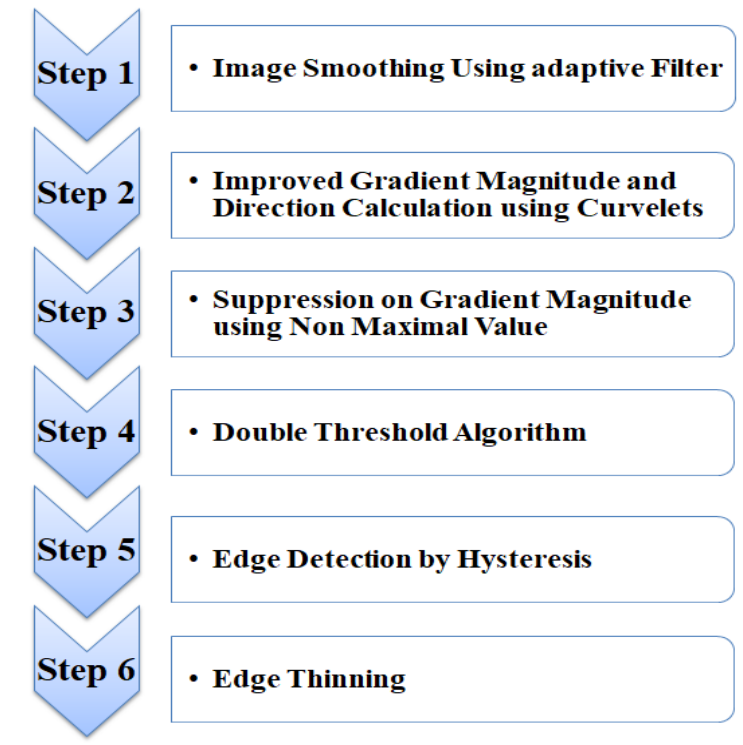

Figure 4: Stepwise Representation of Enhanced Canny Edge Detection Method Input : $\quad$ Gray scale representation of color image.

Method : Adaptive filters are applied and then Selection of two threshold values namely high and low threshold values are used to find the strong or weak edge pixels.

Output : $\quad$ Edge Points. 


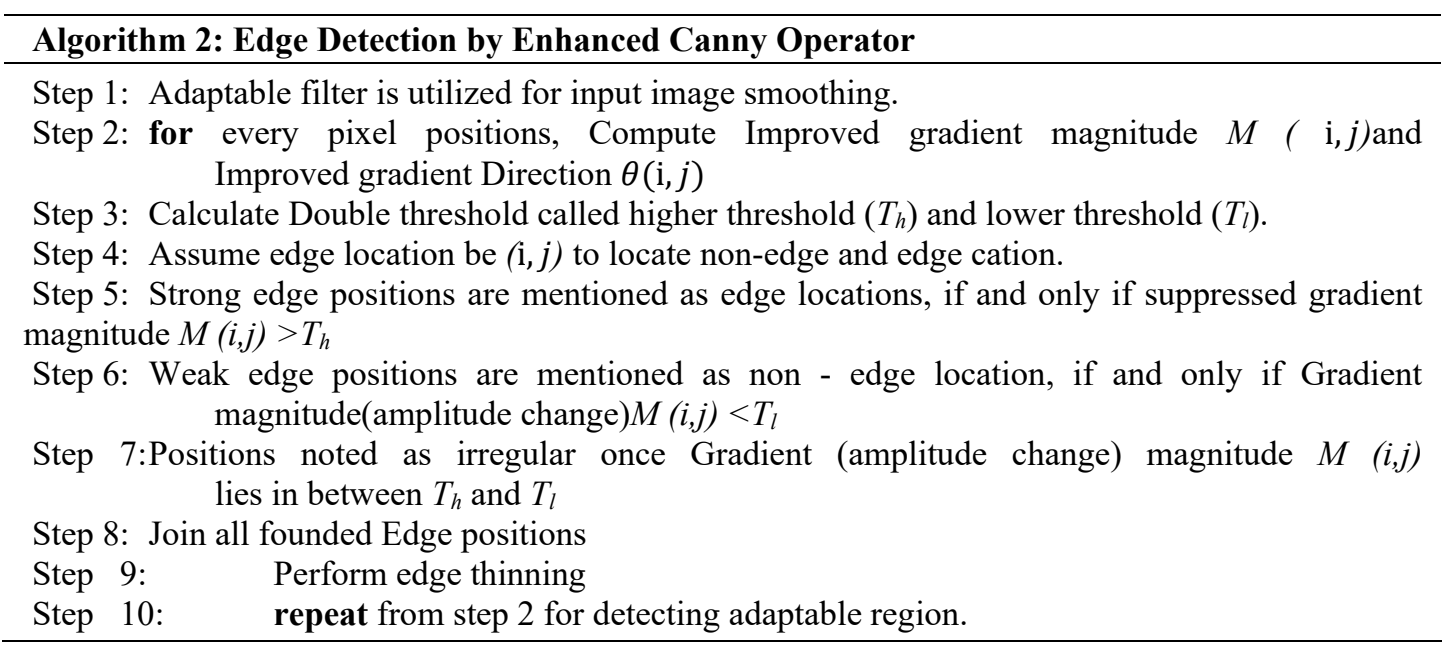

Image Smoothing Using Adaptive Filter.

Smoothing image noise rather than edges are the chief goal of adaptable filters. To accomplish the goal higher weight value is assigned to the filter. Peaking the disjointedness, the lessened the weight is assumed for smoothing. Contrarily, lower the disjointedness between the gray scale levels, higher the weight is assumed for filtering. Adaptable filtration is summarized as follows,

1. For ' $n$ ' iteration and ' $h$ ' be edge amplitude constant with $K=1$.

2. Compute the gradient value $G_{m}(m, \mathrm{n})$ and $G_{n}(m, \mathrm{n})$

3. Estimate the weight by equation 7 and 8:

$$
\begin{gathered}
\mathrm{D}(m, \mathrm{n})=\sqrt{G_{m}(m, n)^{2}+G_{n}(m, n)^{\wedge} 2} \\
\mathrm{~W}(\mathrm{~m}, \mathrm{n})=\exp \left(-\left(\frac{\sqrt{D(m, \mathrm{n})}}{2 h^{2}}\right)\right)
\end{gathered}
$$

4. The adaptive filter is given by equation 9 :

$$
f(m, n)=\frac{1}{N} \sum_{p=-1}^{1} \sum_{q=-1}^{1} f(m+p, n+q) W(m+p, n+q)
$$

5. If $\mathrm{K}$ reaches the last iteration ' $\mathrm{n}$ ' then stop the iteration else increment $\mathrm{K}$ by 1 and proceed from step 2.

\section{Improved Gradient intensity change (Magnitude) and Orientation (Direction) Calculation.}

Improved canny operator for a $3 X 3$ range is concerned with eight levels i.e. horizontally and vertically dual edge points and diagonally two dual edge points. Improved amplitude and orientations are computed as follows in equation (10) and (11)

$$
\begin{gathered}
M_{x}(s, t)=\left[\begin{array}{c}
I(s+1, t+1)+I(s-1, t+1)+I(s+1, t) \\
+I(s, t+1)-I(s-1, t-1) \\
-I(s+1, t-1)-I(s-1, t)-I(s, t-1)
\end{array}\right] / 2 \\
M_{y}(s, t)=\left[\begin{array}{c}
I(s+1, t-1)+I(s+1, t)+I(s+1, t+1) \\
+I(s, t+1)-I(s-1, t-1) \\
-I(s-1, t)-I(s-1, t+1)-I(s, t-1)
\end{array}\right] / 2
\end{gathered}
$$

Gradient amplitude change (magnitude) and orientation (direction) is computed by equation 12 and 13 as follows,

$$
\begin{aligned}
M(s, t) & =\sqrt{\left(\left(M_{x}(s, t)^{\wedge} 2+M_{y}(s, t)^{\wedge} 2\right)\right)} \\
\theta(s, t) & =\arctan \left(M_{x}(i, j) / M_{y}(i, j)\right)
\end{aligned}
$$


For representing the smoothened images, curvelet are the basic concept to identify the perfect edges of the images. Curvelet with greater resolution value is considered to define the attributes of the image.

\section{Suppression, Double Threshold and Edge Detection Algorithm.}

The high threshold value is obtained by applying Otsu's method on non-maximal suppressed image gradient magnitude and direction. Low threshold value is half of the high threshold value. Threshold of dissimilar sets of images find strong edges. Except fixing of the threshold values, the rest of the steps are implemented as it is discussed in previous section.

\section{Thinning of Edge.}

Thinning of edge is to concentrate on weak point edges with high frequency feature. As the weak point edges may get preserved for further processing by comparing the weak edge point pixel with rest of the eight neighbor pixels. If any of the pixel among the eight pixel matches with the weak edge point, then the pixel should be preserved. Local sharpening of the weak edge is done to avoid breaks in the detected boundaries of the image [14].

\subsection{Otsu's Method}

Local adaptive region variance of every pixel is calculated by using the threshold values in the Otsu's method. Computed local adaptive region variance becomes equivalent to the global adaptive region variance (Sezgin et al. 2004). Once the histogram of the gray scale image is computed then Otsu's method can be performed immediately on the histograms of the gray scale values [12]. The method gets directly applied on the histograms values because it is a faster method comparative to other algorithms. Algorithm 3, explains Otsu's Method.

Uniform illumination of the pixel values helps in detecting the adaptive region by assuming the image and the histogram value as bimodal [15]. Behavioral analysis based on the intensity of every pixel is done by considering various intensity levels. The weighted Local Adaptive Region is computed by the sum of products of Local adaptive region probabilities and Local adaptive region variance.

Input : Gray scale representation of color image.

Method : Assume a seed point and the intensity level of the selected seed point gets compared with adjacent pixels by applying adaptive region variance.

Output : Identified adaptive regions for the selected seed points.

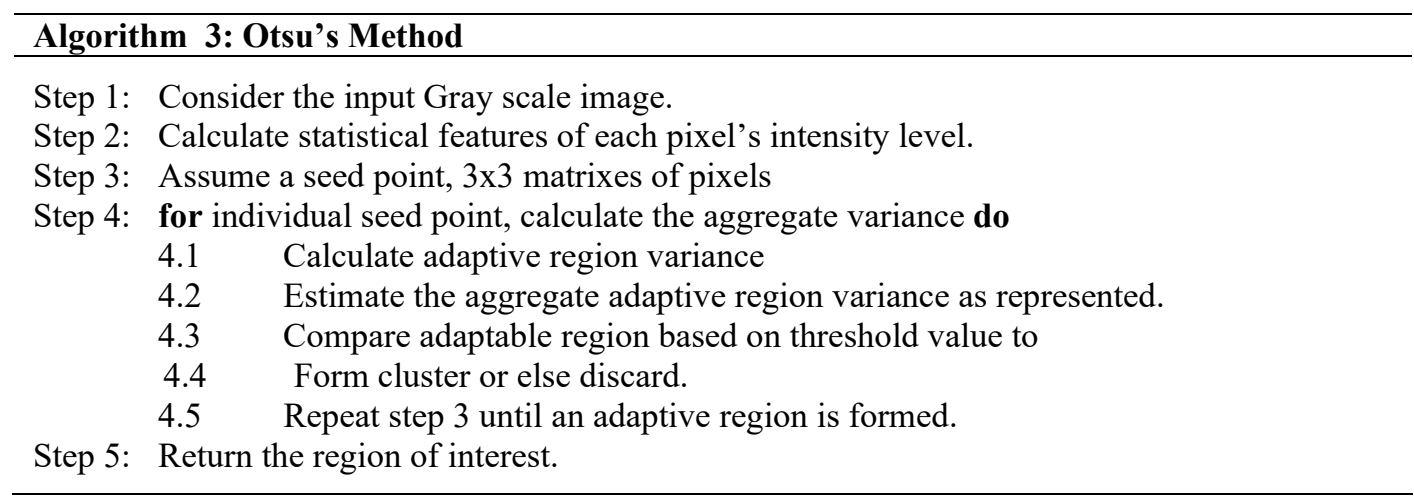

\subsection{Watershed Algorithm}


The Watershed algorithm segments the adaptive regions of the image by using morphology based mathematical operators. Figure 5 explains watershed segmentation algorithm.

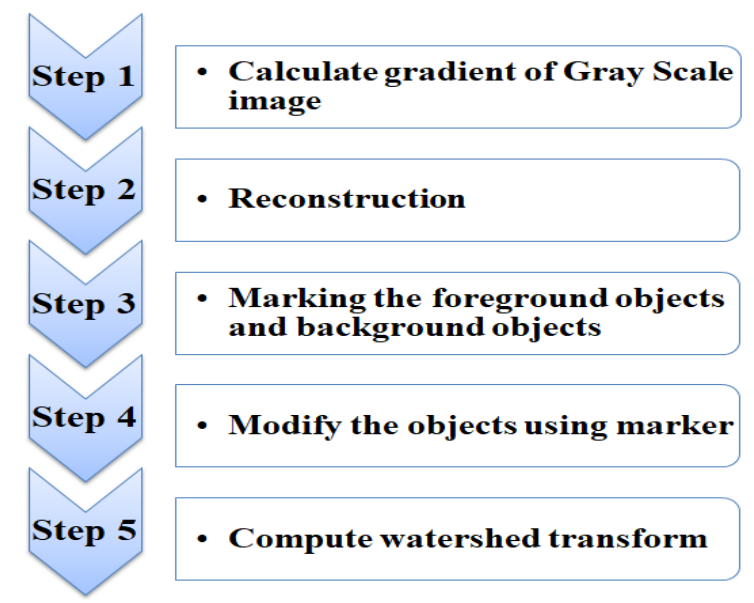

Figure 5: Stepwise Representation of Watershed Algorithm

Gray scale of image gradient is calculated and reconstructed by morphological mathematical operator. In the reconstructed image, compute regional maxima by marking foreground and background objects. Constant intensity ' $t$ ' of the pixels are connected to form a regional maxima and the pixel's intensity outside the boundary is less than $t$. Based on the foreground and background marker, modify the gray scale image gradient. Once the regional minimal of a location is obtained then apply the watershed transformation algorithm.

\subsection{Real Time Adaptive Color Image Segmentation}

The concept of region based image segmentation identifies the region based on the matches found in the color complexity of the pixels. Region based image segmentation has two different approaches and they are,

$$
\text { 1. Split and Merge }
$$

2. Region Growing

\section{Split and Merge Image Segmentation.}

Until a uniform region in the image is obtained, the non-uniform regions are split repeatedly. Maximal uniform region is obtained by merging the subdivided regions further.

\section{Region Growing Based Image Segmentation.}

Seed value is assumed for clustering the region. The neighbor pixel's color complexity is considered for region growing. The absolute region shaped is the adaptive region based on its color. Region growing not only considers the seed point complexity with neighbor pixel but also it compares the neighboring adaptive region for its unions or adaptability of the clusters.

Boundary relaxation based Image segmentation of color images is done. Segmentation can also be agglomerative grouping of analogous neighborhoods. Color image segmentation can be RGB Space based or Watershed Segmentation in region growing technique. Real-time adaptive color image segmentation is an extension lead by Real-time color image segmentation [16]. This real time adaptive image segmentation also works for image steganalysis in finding the hidden information. An 8 by 2-pixel consideration window around every seed point is divided into two unequal sub ranges in 8 different orientations.

Algorithm 4, explains the real time adaptive image segmentation as follows,

Input : Pre-processed Image. 
Method : $\quad$ Comparing seed point with neighboring pixels.

Output : $\quad$ Segmented image based on color adaptability of the seed pixel.

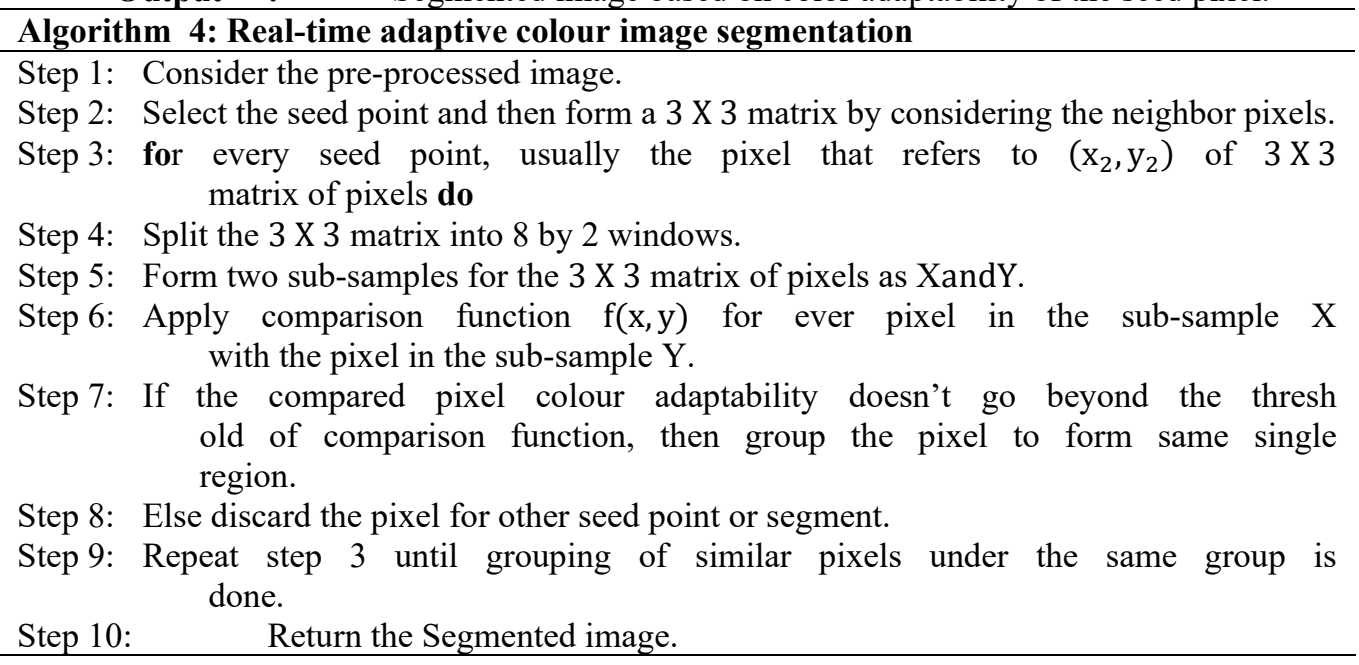

\section{Analysis of the Proposed Adaptive Image Segmentation System}

In this section the performances of the image classification system using image segmentation is discussed. The image segmentation methods such as Enhanced canny edge detection, Canny edge detection, Otsu's method, Watershed method and Real time adaptive color image segmentation used by the proposed system is evaluated in Figure 6 and it describes the identification of adaptive region using various image segmentation methods. From the figure, it is clear that Improved canny operator for detection of edge performs well in Identification of adaptable region.

Though the Watershed process also produces better results comparative to the segmentation methods, it is not considered for implementation because watershed algorithm identifies and splits the region very specifically into minute regions. This minute separation of region decreases the overall performance of region identification and the same is represented in Figure 7 as follows, 


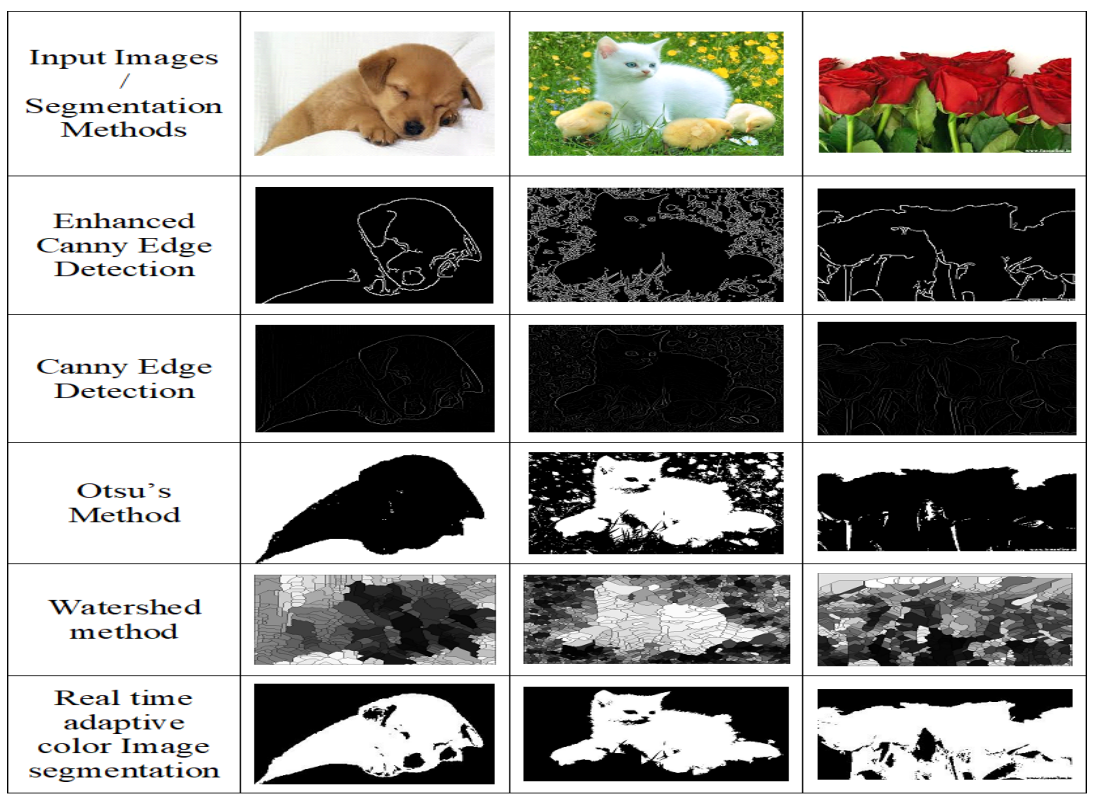

Figure 6: Adaptive Region Identification by Different Segmentation Methods

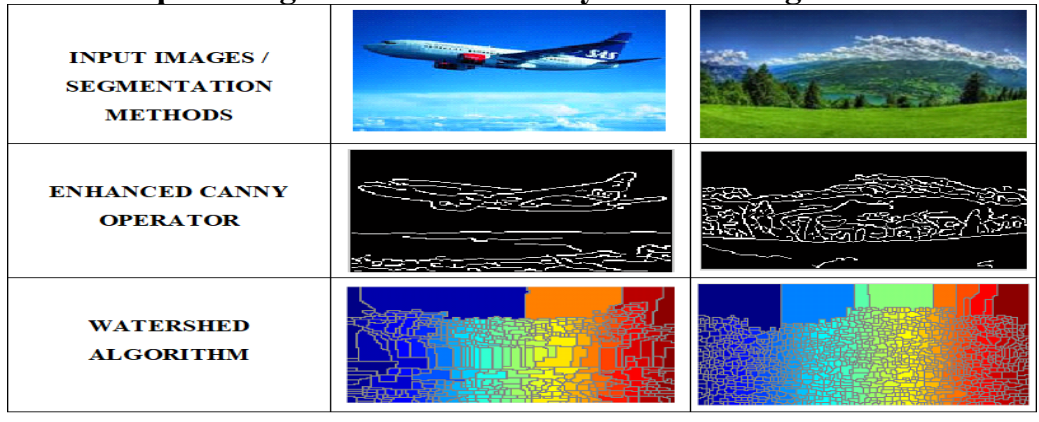

Figure 7 Adaptive Region Identification

The classification accuracy of different image segmentation methods is shown in table 2 and Figure 8. It implies that the proposed enhanced canny edge detection algorithm outperforms the other segmentation methods.

Table 2 Classification Accuracies for Different Segmentation Methods

\begin{tabular}{|l|l|}
\hline Segmentation Method & Classification Accuracy \\
\hline Enhanced Canny Edge Detection & $96.72 \%$ \\
\hline Canny Edge Detection & $95.23 \%$ \\
\hline Otsu's Method & $93.16 \%$ \\
\hline Watershed Method & $96.30 \%$ \\
\hline Real Time Adaptive Color Image Segmentation & $95.10 \%$ \\
\hline
\end{tabular}




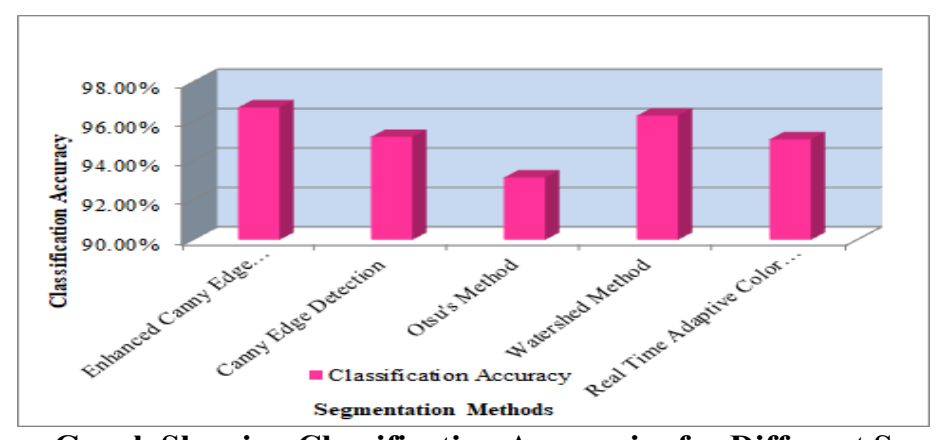

Figure 8: Graph Showing Classification Accuracies for Different Segmentation Methods

\section{Conclusion}

This Conclusion explains about the frame work of the proposed method and the image dataset used. It also investigated five different methods to identify the suitable adaptive color image segmentation method which was otherwise called as enhanced canny edge detection algorithm for giving better input to the next phase called the feature extraction by experimental analysis. This method perfectly segments the Region of Interest from where the features can be extracted for further processing. The first step in enhanced canny edge detection algorithm uses an adaptive filter for image smoothing. The adaptive filter usage helps to include the weak signal points with high frequency into preserved list. Improved gradient magnitude and four gradient directions used to verify the pixel belongs to which cluster. Double threshold called low and high threshold severs as boundaries for selecting the pixel in the range. Edge thinning is local sharpening of the pixel to be considered in preserved list. The experimental analysis results in perfect segmentation by watershed algorithm as like enhanced canny edge detection algorithm. Though the results of watershed algorithm are more accurate for segmentation, the segmentation methodology considered for adaptive image steganalysis is edge identification by enhanced canny operator with $96.72 \%$ of accuracy. The recommended method segments perfect adaptive region. The results imply that the number of pixels in the adaptive region increases above a threshold value worsens the feature extraction accuracy and if within the threshold value the segmentation works perfectly for identifying the adaptive color region of interest.

\section{References}

[1] Dilpreet Kaur and Yadwinder Kaur (2014), "Various Image Segmentation Techniques: A Review", International Journal of Computer Science and Mobile Computing, Vol.3, No. 5, pp. 809-814.

[2] Bhargavi, K. and Jyothi, S. (2014), "A Survey on Threshold Based Segmentation Technique in Image Processing", International Journal of Innovative Research and Development, Vol. 3, No. 12, pp. 234-239.

[3] Muthukrishnan, R. and Radha (2011), "Edge Detection Techniques for Image Segmentation", International Journal of Computer Science and Information Technology (IJCSIT), Vol. 3, No. 6, pp. 259- 267.

[4] Ehsan Nadernejad, Sara Sharifzadeh and Hamid Hassanpour, (2008), "Edge Detection Techniques: Evaluations and Comparisons", Applied Mathematical Sciences, Vol.2, No.31, pp.1507-1520. 
[5] Wang Ran, Xu Man-Kun, Ping Xi-Jian and Zhang tao (2014), "Steganalysis of Spatial Images Based on Segmentation”, ActaAutomaticaSinica, Vol. 40, No. 12, pp. 2936-2943.

[6] Sathya, B. and Manavalan, R. (2011), "Image Segmentation by Clustering Methods: Performance Analysis", International Journal of Computer Applications, Vol. 29, No. 11, pp.27-32.

[7] Amandeep Kaur and Aayushi (2014), "Image Segmentation Using Watershed Transform", International Journal of Soft Computing and Engineering, Vol. 4, No. 1, pp.5-8.

[8] Tan, J. and Yahya, A. A. (2012), "A Novel Partial Differential Equation Method Based on Image Features and It's Applications in Denoising", 4th International Conference on Digital Home (ICDH), pp. 46-51.

[9] Sujatha, P., Purushothaman, S. and Rajeswari, R. (2014), "Performance Study of Combined Artificial Neural Network Algorithms for Image Steganalysis", Proceedings of International Conference on Internet Computing and Information Communications, Part of the Advances in Intelligent Systems and Computing, Vol. 216, pp. 441-451.

[10] Shiuh-Yung Chen, Wei-Chung Lin and Chin-Tu Chen (1991), "Split-and-merge image segmentation based on localized feature analysis and statistical tests", CVGIP: Graph. Models Image Process, Vol. 53, No. 5, pp. 457-475.

[11] Wu, ZY (1993), "Homogeneity testing for unlabeled data: A performance evaluation", CVGIP: Graph. Models Image Process, Vol. 55, No. 5, pp. 370-380.

[12] Ross Beveridge, J., Joey Griffith, Ralf R. Kohler, Allen R. Hanson and Edward M. Riseman (1989), "Segmenting images using localized histograms and region merging", International Journal of Computer Vision, Vol. 2, No. 3, pp. 311-347.

[13] Ping Zhou, Wenjun, Y.E. and Yaojie Xia and Qi Wang (2009), "An Improved Canny Algorithm for Edge Detection”, International Journal of Computational Information System, Vol.7, No. 5, pp. 1516-1523.

[14] Mallat, S. and Zhong, S. (1992), "Characterization of Signals from Multi scale Edges”, IEEE Trans on PAMI, Vol. 14, No. 7, pp. 710-732.

[15] Zhang, T.X., Peng, J.X. and Li, Z.J. (1996),“An Adaptive Image Segmentation Method with Visual Nonlinearity Characteristics", IEEE Trans. on SMC-Part B, Vol. 26, No. 4, pp. 619 -627.

[16] Ramakant Nevatia (1977), “A Color Edge Detector and Its Use in Scene Segmentation”, IEEE Transactions on Systems, Man, and Cybernetics, Vol. 7, No.11, pp. 820-826. 